\title{
Intraoperative neuromonitoring alerts in a pediatric deformity center
}

\author{
Maria Zuccaro, PhD, CNIM, James Zuccaro, DC, DABNM, ${ }^{1}$ Amer F. Samdani, MD, ${ }^{2}$ \\ Joshua M. Pahys, MD, ${ }^{2}$ and Steven W. Hwang, MD²
}

Departments of ${ }^{1}$ Intraoperative Neuromonitoring and ${ }^{2}$ Surgery, Shriners Hospitals for Children-Philadelphia, Pennsylvania

OBJECTIVE Intraoperative neuromonitoring (IONM) involves the use of somatosensory evoked potentials (SSEPS) and transcranial electric motor evoked potentials (TceMEPs). In this retrospective study the authors examined the sensitivity and specificity of both SSEPs and TceMEPs during pediatric spinal deformity surgeries.

METHODS The authors performed a retrospective quantitative analysis of data obtained in 806 patients (197 males and 609 females) treated from December 2011 until October 2015. All patients were diagnosed with scoliosis that was classified as one of the following: adolescent idiopathic scoliosis (AIS) (38\%), congenital scoliosis (22\%), or syndromic scoliosis (40\%). Also, 53 patients underwent vertebral column resection (VCR). All surgeries were monitored by high-level neuromonitoring specialists and were performed with total intravenous anesthesia. Alerts were described as a decrease in amplitude by $50 \%$ or greater (bilateral or unilateral) in SSEPs, TceMEPs, or both.

RESULTS True-positive alerts for TceMEPs were observed in 60 of the 806 patients (7.4\%). True-positive alerts for SSEPs were observed in 7 of the 806 patients $(0.9 \%)$. In contrast, there were no false-positive or false-negative outcomes. Only 1 case $(0.1 \%)$ was reported with a permanent postoperative deficit. No reported false negatives or false positives were observed, and thus sensitivity was $100 \%$ and specificity was $93 \%-100 \%$ for TceMEPs. The rate of sensitivity was $13.2 \%$ and the rate of specificity was $100 \%$ for SSEPs. The breakdown of total alert was as follows: $6.6 \%$ in AIS cases, $24.5 \%$ in congenital scoliosis cases, and $10.2 \%$ in syndromic scoliosis cases. Neurological injury rates were significantly lower than in previous studies, as there were $0 \%$ for AIS cases $(p=0.12), 0.6 \%$ for congenital scoliosis cases $(p=0.17)$, and $0 \%$ for syndromic scoliosis cases $(p=0.07)$. One injury in a patient with congenital scoliosis occurred during a VCR procedure, which brought the injury rate to $1.9 \%(p<0.005)$. IONM alerts occurred during $34 \%$ of rod/correction cases, $25 \%$ of thoracic screw placements, $20 \%$ of the osteotomies, $17 \%$ of the resections, $3 \%$ of the cage insertions, and $2 \%$ of the sublaminar wiring procedures.

CONCLUSIONS The authors hypothesize that the results of this study will support the necessity, as a standard of care, of multimodality neuromonitoring during high-risk pediatric spinal deformity surgery because of the decrease in postoperative deficits. Their data suggest that the TceMEPs are more sensitive than SSEPs, but when used in combination, they offer the patient a level of safety that would otherwise not exist. Last, these findings support the notion that better outcomes are achieved with high-level IONM professionals.

https://thejns.org/doi/abs/10.3171/2017.7.FOCUS17364

KEY WORDS intraoperative neuromonitoring; transcranial electric motor evoked potential; somatosensory evoked potential

I $\mathrm{N}$ the United States, the vast majority of pediatric deformity surgeries are performed while using intraoperative neuromonitoring (IONM). If reliable transcranial electric motor evoked potentials (TceMEPs) are present, they can be used intraoperatively as a substitute for performing the wake-up test. ${ }^{1}$ However, multiple factors may influence the ability to generate baseline evoked potentials in certain pediatric populations..$^{1,2,12}$ Because of their associated risks of complete or partial paralysis, surgical procedures that may benefit from the use of evoked potential monitoring include the initial placement of both expandable spinal rods and vertical expandable prosthetic titanium rods (VEPTRs), the initial lengthening of both rod types, and vertebral column resections. ${ }^{1,2}$

ABBREVIATIONS IONM = intraoperative neuromonitoring; PPV = positive predictive value; $\mathrm{RSC}=$ reversible spinal cord change; SSEP = somatosensory evoked potential; TceMEP = transcranial electric motor evoked potential; TIVA = total intravenous agent; VEPTR = vertebral expandable prosthetic titanium rib. ACCOMPANYING EDITORIAL DOI: 10.3171/2017.7.FOCUS17457.

SUBMITTED June 1, 2017. ACCEPTED July 5, 2017.

INCLUDE WHEN CITING DOI: 10.3171/2017.7.FOCUS17364. 
Certain variables may influence the ability to generate baseline evoked potentials, including the patient's chronological age, cognitive ability, and a history of neurological disorders. ${ }^{1}$ With regard to a patient's chronological age, the current literature suggests neuronal pathways and the myelination of these pathways do not fully develop until after the age of 2 years. ${ }^{1}$ Therefore, obtaining evoked potentials in this young population poses a great challenge for the neurophysiology professional. However, both somatosensory evoked potentials (SSEPs) and TceMEPs are deemed safe for this population, and by all means monitoring should be attempted prior to incision. ${ }^{1}$ Also, cognitive ability has been associated with obtaining baseline

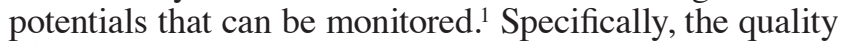
of the cortex being stimulated may determine the robustness of baseline data. ${ }^{1}$ Last, neurological disorders, such as seizures, were once thought to be a contraindication to TceMEPs. ${ }^{1-3,8}$ According to MacDonald and colleagues, the incidence of seizures was $0.03 \%$ and the incidence of bite injuries was $0.2 \% .^{6}$ Therefore, a risk/benefit analysis should be performed prior to utilizing TceMEPs.

For over 20 years, surgeons have had access to modalities such as TceMEPs and SSEPs during their surgical procedures. ${ }^{5,12}$ In high-risk surgeries, such as those used in spinal deformity correction (i.e., scoliosis reduction) a $0.3 \%-0.8 \%$ chance of a postoperative deficit has been observed. ${ }^{5}$ Multimodality neuromonitoring of SSEPs and TceMEPs allows for a greater level of specificity regarding patient outcome after spinal deformity surgery. ${ }^{2}$ Nuwer et al. observed a sensitivity level of $92 \%$ and a specificity level of $98 \%$ for SSEP monitoring, in terms of new postoperative motor deficits. ${ }^{7}$ SSEP monitoring evaluates the integrity of the dorsal column's medial lemniscal pathway. ${ }^{2}$ The dorsal columns are located in the posterior two-thirds of the spinal cord, and blood is supplied by 2 posterior spinal arteries. ${ }^{4}$ Three limitations of SSEP monitoring exist. First, the small computerized averaged response lacks immediate feedback through data collection. ${ }^{2}$ Second, SSEPs do not reveal the integrity of motor tracts. ${ }^{2}$ Third, there may be a delay in response time. The current literature suggests that such a lag time may be 5 to 16 minutes. ${ }^{3}$

In contrast, TceMEP monitoring allows for evaluation of motor function through electrical stimulation of the cerebral cortex, and also for evaluation of the integrity of the anterolateral cortical spinal tracts. ${ }^{2}$ Additionally, this tract is located in the anterior third of the spinal cord, and the blood is supplied through the anterior spinal artery. ${ }^{5}$ Moreover, because this tract is rich in cell bodies and synapses, it is more metabolically active. ${ }^{5}$ These factors suggest that monitoring TceMEPs can provide greater sensitivity for detecting ischemic changes to the spinal cord. ${ }^{5}$ Optimal recording sites for TceMEPs may include the abductor pollicis, abductor longus, and other long extensor/flexor muscles. These muscles are the preferred recording sites because of their rich corticospinal tract innervation within the cortex. ${ }^{2}$ However, the sensitivity of TceMEPs may be influenced by both hypoperfusion and hypothermia, and an effort to control these factors should be attempted. ${ }^{2}$ Moreover, the use of total intravenous agents (TIVAs) and a multipulse stimulation train allow for a greater advantage in overcoming anesthetic agents. ${ }^{4}$ Motor function may be monitored using TceMEPs, but TceMEP monitoring is sensitive to inhalation agents. ${ }^{10}$ Therefore, a TIVA anesthetic protocol is an optimal choice for maintaining sedation throughout the procedure. ${ }^{4,10}$ Also, TceMEP monitoring appears to be more beneficial in detecting a change in spinal cord perfusion to the motor tracts that results from surgical manipulation. The most logical explanation for this finding can be supported by the vascular anatomy of the spinal cord. Again, the anterior spinal artery is responsible for the motor tracts located in the anterior two-thirds of the cord. In contrast, 2 posterior spinal arteries supply the sensory tracts located in the posterior aspect of the cord. Moreover, the anterior portion of the cord contains a large number of cell bodies and synapses that are highly sensitive to ischemic changes. ${ }^{5}$ Last, the anterior portion of the cord has greater metabolic demands and fewer radicular arteries than the posterior portion of the cord. ${ }^{5}$ All of these factors suggest an increased sensitivity to ischemic changes that can be detected through TceMEP monitoring. ${ }^{5}$

Although the benefits of multimodality monitoring are positive, some institutions still do not use this approach in pediatric spinal surgeries. This paper demonstrates the benefits of a multimodality approach and reports the levels of specificity that TceMEP and SSEP monitoring have achieved at our institution, which is a regional spinal deformity center.

\section{Methods}

After obtaining approval from Temple University's Institutional Review Board, we performed a retrospective review of the neuromonitoring and surgical records; the search yielded 809 patients who had undergone scoliosis surgery in which neuromonitoring was used during the period from December 2011 until October 2015. Both male and female patients were examined, and ages ranged from 1 to 20 years. Patients excluded from the study were those with a preexisting spinal cord injury or preexisting lower-extremity paralysis. Patients included in the study population had one of the following diagnoses: idiopathic, congenital, or syndromic scoliosis, which included neuromuscular and various syndromes. A data entry system was created for these patients' cases and included the following variables: diagnosis, TceMEP alerts, SSEP alerts, surgical or anesthetic interventions, and time required for the alert to resolve. Alerts were defined as a drop of $50 \%$ in amplitude of either TceMEPs or SSEPs. In addition, an alert needed to be accompanied by a surgical event that posed a risk of spinal cord trauma (e.g., screw placement, osteotomy, rod correction, or derotation).

Continuous neurophysiological monitoring was performed from the time the patient was positioned until 30 minutes after the last major corrective force was applied to the rods that were placed. Both SSEPs and TceMEPs were monitored using a Cascade Elite neuromonitoring system (Cadwell). Potentials were recorded and interpreted by a group of 2 nationally certified trained individuals who had successfully completed multiple graduate-level neuroanatomy/physiology educational courses and who were present throughout the entire procedure. All IONM changes were 
TABLE 1. Patient and surgical procedure data

\begin{tabular}{|c|c|c|c|c|c|c|c|c|c|}
\hline \multirow[b]{2}{*}{ Surgical Procedure } & \multirow{2}{*}{$\begin{array}{l}\text { Total No. of } \\
\text { Patients Monitored }\end{array}$} & \multicolumn{2}{|c|}{ Sex } & \multicolumn{2}{|c|}{ Age (yrs) } & \multicolumn{2}{|c|}{ Cobb Angle $\left(^{\circ}\right)$} & \multirow{2}{*}{$\begin{array}{l}\text { SSEPs } \\
\text { Obtained }\end{array}$} & \multirow{2}{*}{$\begin{array}{l}\text { TceMEPs } \\
\text { Obtained }\end{array}$} \\
\hline & & Male & Female & Range & Mean & Range & Mean & & \\
\hline Overall no. of PSFs & 511 & 128 & 383 & $1-20$ & 13.8 & $40-160$ & 71.7 & 436 & 498 \\
\hline For AIS & 136 & 28 & 108 & $6-20$ & 14.0 & $40-134$ & 63.8 & 132 & 136 \\
\hline For congenital scoliosis & 53 & 12 & 41 & $1-19$ & 11.5 & $38-120$ & 76.6 & 46 & 53 \\
\hline For syndromic scoliosis & 322 & 88 & 234 & $5-20$ & 13.8 & $42-160$ & 84.0 & 258 & 309 \\
\hline VCR & 52 & 16 & 36 & $1-20$ & 12.3 & $42-150$ & 90.6 & 39 & 50 \\
\hline \multicolumn{10}{|l|}{ Other procedures } \\
\hline VEPTR & 68 & 26 & 42 & $3-13$ & 8.0 & $30-130$ & 77.0 & 59 & 66 \\
\hline Growing rod & 59 & 22 & 37 & $2-13$ & 8.0 & $30-121$ & 80.6 & 46 & 58 \\
\hline VBS & 41 & 7 & 34 & $5-19$ & 11.6 & $8-51$ & 31.5 & 41 & 41 \\
\hline Growth modulation & 127 & 14 & 113 & $9-15$ & 12.0 & $27-70$ & 45.4 & 127 & 127 \\
\hline
\end{tabular}

AIS = adolescent idiopathic scoliosis; PSF = posterior spinal fusion; VBS = vertebral body stapling; VCR = vertebral column resection.

December 2011 to October 2015. Patient data included surgical procedure, TceMEP alerts, SSEP alerts, surgical intervention, anesthetic intervention, and time until recovery of evoked potentials. A significant IONM change was defined as a loss of $50 \%$ of amplitude of either TceMEPs or SSEPs coinciding with surgical interventions. Neuromonitoring alerts were determined by the aforementioned criteria and then categorized utilizing Bradford Hill causality guidelines; Skinner and Sala determined that using causality guidelines can help determine a true-positive change based on the causal relationship between the intervention and recovery of the evoked response. ${ }^{10}$ Therefore, a true-positive change was defined as a decrease in amplitude of response based on the alert criteria, correlated with a surgical event, which either recovered with intervention or which remained at closing and correlated with a postoperative neurological impairment seen upon the patient's emergence from anesthesia. A false-positive change was defined as a loss of amplitude that did not return at closing, yet the patient emerged from anesthesia with no new neurological impairment. A true-negative change was defined as no loss of amplitude and no new neurological impairments upon emergence from anesthesia. Finally, a false-negative result was defined as no loss of amplitude at closing and a new postoperative neurological impairment. According to Skinner and Holdefer, reversible spinal cord changes (RSCs) are typically classified as true-positive IONM alerts, but because they are corrected by intervention, it is difficult to determine if these events were real IONM changes without a true finding of a correlating neurological deficit. ${ }^{11}$ With this in mind, it is suggested that a range be calculated for specificity and positive predictive value (PPV). Three groups were used: in the first, all RSCs were considered true positives; in the second, all
RSCs were all considered false positives; and in the third, causality guidelines were applied to the RSCs to classify them. For each group, specificity and PPV were calculated.

\section{Results}

A multimodality approach to neuromonitoring was used in all patients; patient and surgical procedure data are presented in Table 1 . The total number of patients examined was 806 (197 male and 609 female). Ages ranged from 1 to 20 years (median 12.5 years). Cobb angles ranged from $21^{\circ}$ to $180^{\circ}$ (median $66.2^{\circ}$ ). Of the 806 patients, 511 underwent a posterior spinal fusion. The posterior spinal fusions were categorized into adolescent idiopathic $(n=136)$, congenital $(n=53)$, and syndromic $(n=322)$ scoliosis; also, 52 patients underwent vertebral column resection. Additional spinal procedures consisted of VEPTR $(n=68)$, growing rod $(n=59)$, vertebral body stapling $(n=41)$, and growth modulation $(\mathrm{n}=127)$. TceMEP and SSEP monitoring were attempted pre-incision in all 806 patients.

For the 806 patients in the study population, the number of true-positive TceMEP alerts was 60, and the number of true-positive SSEP alerts was 7 (Table 2). In contrast, no reported false-positive or false-negative outcomes were observed. Additionally, there was 1 reported postoperative deficit. Sensitivity and specificity rates for TceMEP alerts were both $100 \%$. In the above calculations, it was assumed that all RSCs were in fact true positives. To determine a range, the RSCs were broken into 3 groups. In the first group, assuming all RSCs were indeed true-positive TceMEP alerts, specificity was calculated to be $100 \%$, with a PPV of 1. In the second group, assuming all RSCs were false-positive TceMEP alerts, specificity was $93 \%$, with a PPV of 0.08 . In the third group, applying causality guide-

TABLE 2. IONM alert totals

\begin{tabular}{|c|c|c|c|c|c|c|c|c|c|c|}
\hline \multirow{2}{*}{$\begin{array}{l}\text { No. of } \\
\text { Cases }\end{array}$} & \multicolumn{2}{|c|}{ True-Pos Alerts } & \multicolumn{3}{|c|}{ Other Alerts } & \multirow{2}{*}{$\begin{array}{l}\text { Postop } \\
\text { Deficit }\end{array}$} & \multicolumn{2}{|c|}{ TceMEPs (\%) } & \multicolumn{2}{|c|}{ SSEPS (\%) } \\
\hline & TceMEPs & SSEPS & True Neg & False Pos & False Neg & & Sensitivity & Specificity & Sensitivity & Specificity \\
\hline 806 & 60 & 7 & 746 & 0 & 0 & 1 & 100.00 & $93-100$ & 13.21 & 100.00 \\
\hline
\end{tabular}

Neg = negative; pos $=$ positive . 


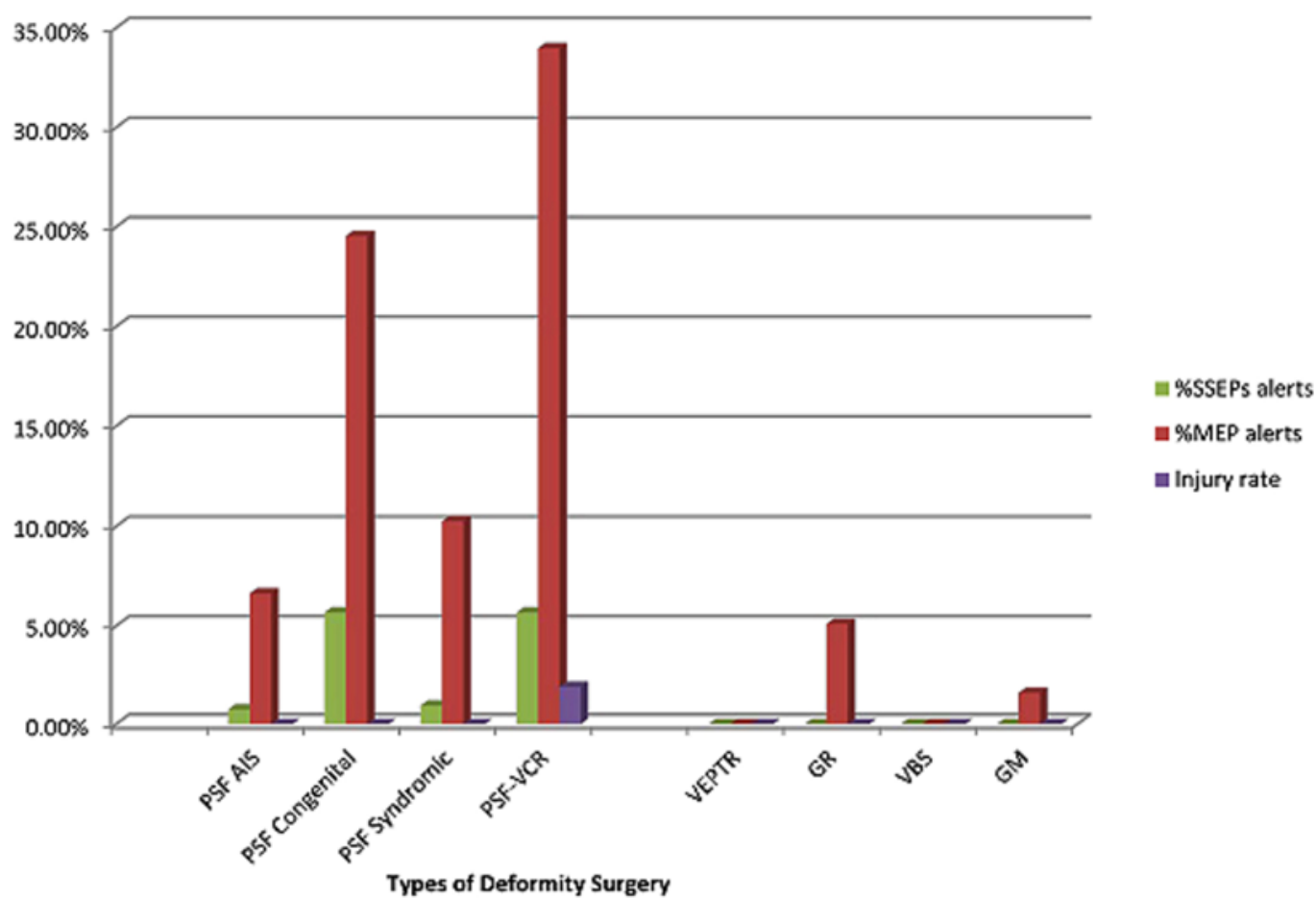

FIG. 2. Bar graph showing neuromonitoring data according to type of surgical procedure. AIS = adolescent idiopathic scoliosis; $\mathrm{GM}=$ growth modulation; $\mathrm{GR}$ = growing rod; $\mathrm{MEP}=$ motor evoked potential; $\mathrm{PSF}$ = posterior spinal fusion; VBS = vertebral body stapling; VCR = vertebral column resection.

lines to RSCs, specificity was $100 \%$, with a PPV of 0.98 . Thus, the specificity range for TceMEP alerts was $93 \%$ to $100 \%$. In comparison, the sensitivity and specificity for SSEP alerts were 13.21 and $100 \%$, respectively. IONM alert data according to surgery type are presented in Fig. 2 and Table 3.

The surgical causes for each neuromonitoring alert were thoracic screw placement $(25 \%)$, osteotomy $(20 \%)$, cage placement $(3 \%)$, wiring $(2 \%)$, resection $(17 \%)$, and rod/correction (34\%) (Fig. 3). Neurological deficit rates were determined to be as follows: idiopathic fusion, $0 \%$; congenital fusion, $0 \%$; syndromic fusion, $0 \%$; and vertebral column resection, $1.9 \%$ (Table 4, Fig. 4).

\section{Discussion}

Multimodality monitoring that uses both SSEPs and TceMEPs has been shown to increase the sensitivity of this monitoring. ${ }^{4}$ This paper demonstrates the benefits of using the multimodality approach in severe spinal deformity surgery in a pediatric population. Through the use of this type of monitoring by well-trained and educated neurophysiology professionals, only 1 postoperative deficit was reported out of 806 cases studied; this isolated case occurred during a high-risk procedure involving a vertebral column resection, and interventions were performed by the surgical team at the time of TceMEP loss. The re-

TABLE 3. Summary of IONM data

\begin{tabular}{|c|c|c|c|c|c|c|c|c|c|c|c|}
\hline \multirow[b]{2}{*}{ Surgical Procedure } & \multirow{2}{*}{$\begin{array}{l}\text { Total } \\
\text { No. }\end{array}$} & \multicolumn{2}{|c|}{ True-Positive Alert } & \multicolumn{3}{|c|}{ Other Alerts } & \multirow{2}{*}{$\begin{array}{l}\text { Postop } \\
\text { Deficit }\end{array}$} & \multicolumn{2}{|c|}{ TceMEPs (\%) } & \multicolumn{2}{|c|}{ SSEPs (\%) } \\
\hline & & TceMEPs & SSEPS & True Neg & False Pos & False Neg & & Sensitivity & Specificity & Sensitivity & Specificity \\
\hline Overall no. of PSFs & 511 & 55 & 7 & 456 & 0 & 0 & 0 & 100.00 & $93-100$ & 14.58 & 100.00 \\
\hline For AIS & 136 & 9 & 1 & 127 & 0 & 0 & 0 & 100.0 & $93-100$ & 12.5 & 100.0 \\
\hline For congenital scoliosis & 53 & 13 & 3 & 40 & 0 & 0 & 0 & 100.0 & $93-100$ & 30.0 & 100.0 \\
\hline For syndromic scoliosis & 322 & 33 & 3 & 289 & 0 & 0 & 0 & 100.0 & $93-100$ & 10.0 & 100.0 \\
\hline VCR & 53 & 18 & 3 & 35 & 0 & 0 & 1 & 100.0 & $93-100$ & 20.0 & 100.0 \\
\hline \multicolumn{12}{|l|}{ Other procedures } \\
\hline VEPTR & 68 & 0 & 0 & 68 & 0 & 0 & 0 & 100.0 & 100.0 & 100.0 & 100.0 \\
\hline Growing rod & 59 & 3 & 0 & 56 & 0 & 0 & 0 & 100.0 & $93-100$ & 0.0 & 100.0 \\
\hline VBS & 41 & 0 & 0 & 41 & 0 & 0 & 0 & 100.0 & 100.0 & 100.0 & 100.0 \\
\hline Growth modulation & 127 & 2 & 0 & 125 & 0 & 0 & 0 & 100.0 & $93-100$ & 0.0 & 100.0 \\
\hline
\end{tabular}




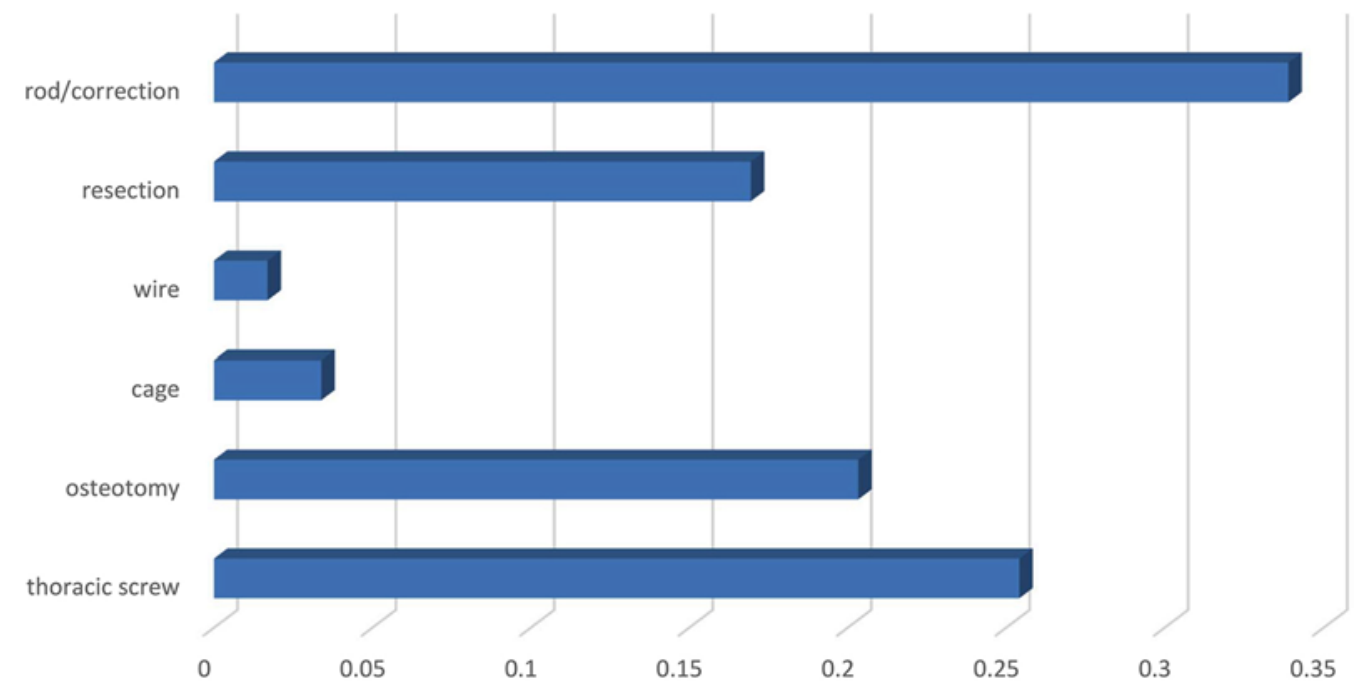

FIG. 3. Surgical events causing neuromonitoring alerts, with percentage of alerts on the $x$ axis and type of surgical event on the $y$ axis.

sults of this study indicate that TceMEP monitoring had a sensitivity of $100 \%$ and a specificity ranging from $93 \%$ to $100 \%$ in detecting spinal cord injury. We also demonstrated that TceMEPs were more sensitive than SSEPs (13.21\% sensitivity) in detecting spinal cord injury. Therefore, our findings suggest that TceMEP monitoring during pediatric spinal deformity surgery can significantly reduce the risk of postoperative deficits. Furthermore, TceMEPs are often used for high-risk spinal deformity surgery, and this is consistent with previous research. ${ }^{1,4}$

Unfortunately, a non-uniform system exists within the field of IONM. Variables that make IONM non-uniform may include the following: use of inhalational agents, slow-charge TceMEP stimulators, data not being interpreted in the room at the time of data loss, and a lack of standard criteria by which to identify and classify an alert. In our study, anesthesia was performed by a consistent group and was strictly managed throughout the procedure. The population base at our institution is often composed of patients who have multiple syndromes or cognitive im-

TABLE 4. IONM alert and injury rate data

\begin{tabular}{lrccc}
\hline \multicolumn{1}{c}{ Surgical Procedure } & $\begin{array}{c}\text { Total } \\
\text { No. }\end{array}$ & $\begin{array}{r}\text { TceMEP } \\
\text { Alerts (\%) }\end{array}$ & $\begin{array}{c}\text { SSEP } \\
\text { Alerts (\%) }\end{array}$ & $\begin{array}{c}\text { Injury } \\
\text { Rate }(\%)\end{array}$ \\
\hline Posterior spinal fusion & & & & \\
\hline$\quad$ For AIS & 136 & 6.6 & 0.7 & 0.0 \\
\hline For congenital scoliosis & 53 & 24.5 & 5.7 & 0.0 \\
\hline$\quad$ For syndromic scoliosis & 322 & 10.2 & 0.9 & 0.0 \\
\hline VCR & 53 & 34.0 & 5.7 & 1.9 \\
\hline Other procedures & & & & \\
\hline$\quad$ VEPTR & 68 & 0.0 & 0.0 & 0.0 \\
\hline Growing rod & 59 & 5.1 & 0.0 & 0.0 \\
\hline VBS & 41 & 0.0 & 0.0 & 0.0 \\
\hline Growth modulation & 127 & 1.6 & 0.0 & 0.0 \\
\hline
\end{tabular}

pairments. Therefore, the use of inhalational agents may not allow for maximum data to be collected due to a lack of quality cortical matter, physiological abnormalities, and immature neurological pathways. ${ }^{1}$ A TIVA protocol was used in all 806 cases, and neuromuscular blockade was only administered for intubation and exposure. The level of neuromuscular blockade was measured using the trainof-four technique from both the ulnar and posterior tibial nerves. Additionally, all 4 twitches returned after exposure was complete. The level of blockade was monitored carefully, communicated clearly to the surgeons and anesthesia team, and documented frequently by the neurophysiology professional in the room. This allowed for in-the-room interpretation by the monitoring individual, ensuring that interventions were performed in a timely manner. No remote monitoring was involved in any of the 806 cases. To our knowledge, this model of IONM professionals is one of the few of its kind in the United States. More impressive is the ability of this team to generate TceMEPs in $98 \%$ of patients and to have a TceMEP-related specificity of 93\%$100 \%$ and a sensitivity of $100 \%$. Historically, this was the predominant IONM practice model prior to the mid- to late 1990s, when vendor remote models started to appear due to financial incentives. According to Skinner and Sala, surgeons should no longer accept "remote" monitoring as it is practiced in the United States. ${ }^{10}$ Our model is consistent with that used in Canada and Europe, and it is a more cost-effective model for any hospital that requires neuromonitoring for its surgical procedures.

Limitations of this study are that it was performed at a single pediatric institution, which means that its findings may not be applicable to an adult center. This study is also limited by its retrospective design. Although all IONM data, surgical procedures, and immediate postoperative evaluations were performed by only 2 IONM professionals, long-term outcomes were subject to postoperative chart review. Also, IONM was conducted by professionals who had significant expertise in pediatrics, and the results of this study may not be able to be extrapolated to centers 


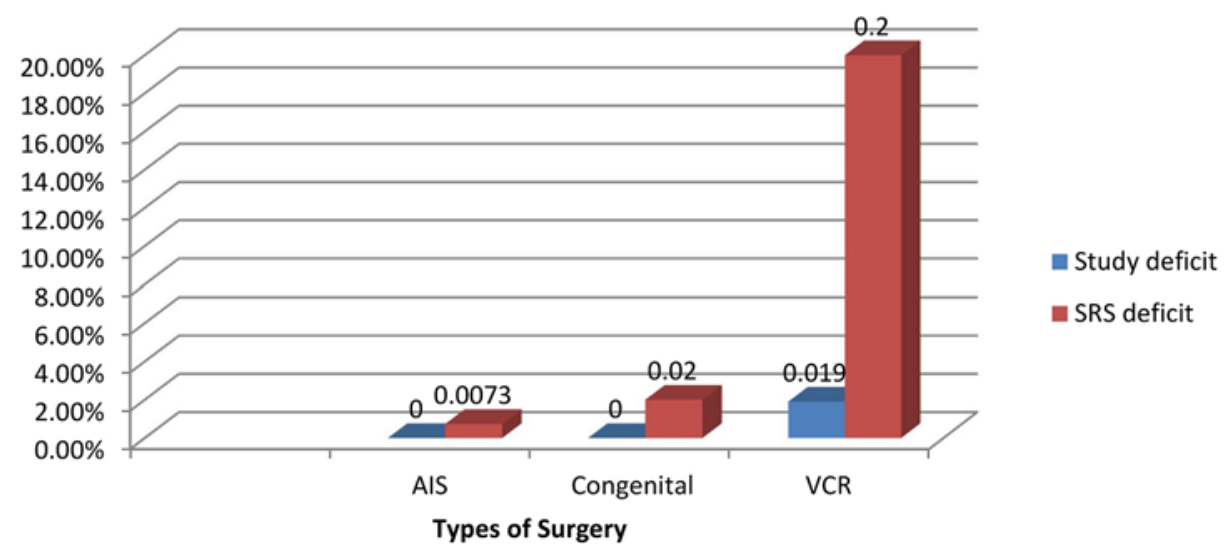

FIG. 4. Bar graph showing neurological injury rates for posterior spinal fusions. SRS = Scoliosis Research Society.

whose monitoring staff does not have this level of expertise.

\section{Conclusions}

The authors hypothesize that the results of this study will support the necessity, as a standard of care, of multimodality neuromonitoring during high-risk pediatric spinal deformity surgery due to the decrease in postoperative deficits. Their findings suggest that TceMEP monitoring is more sensitive than SSEP monitoring, but when used in combination they offer the patient a level of safety that would otherwise not exist. Last, these findings support the notion that better outcomes are achieved with high-level IONM professionals.

\section{References}

1. Emans JB: Intraoperative neuromonitoring applications and issues in pediatric spinal deformity surgery. Spine Deform [epub ahead of print], 2012

2. Ferguson J, Hwang SW, Tataryn Z, Samdani AF: Neuromonitoring changes in pediatric spinal deformity surgery: a single-institution experience. J Neurosurg Pediatr 13:247-254, 2014

3. Hilibrand AS, Schwartz DM, Sethuraman V, Vaccaro AR, Albert TJ: Comparison of transcranial electric motor and somatosensory evoked potential monitoring during cervical spine surgery. J Bone Joint Surg Am 86-A:1248-1253, 2004

4. Hwang SW, Malhotra NR, Shaffrey CI, Samdani AF: Intraoperative neurophysiological monitoring in spine deformity surgery. Spine Deform [epub ahead of print], 2012

5. Koht A, Sloan TB, Toleikis JR (eds): Monitoring the Nervous System for Anesthesiologists and Other Health Care Professionals. New York: Springer, 2012

6. Macdonald DB, Skinner S, Shils J, Yingling C: Intraoperative motor evoked potential monitoring - a position statement by the American Society of Neurophysiological Monitoring. Clin Neurophysiol 124:2291-2316, 2013

7. Nuwer MR, Dawson EG, Carlson LG, Kanim LE, Sherman JE: Somatosensory evoked potential spinal cord monitoring reduces neurologic deficits after scoliosis surgery: results of a large multicenter survey. Electroencephalogr Clin Neurophysiol 96:6-11, 1995

8. Schwartz DM, Sestokas AK, Dormans JP, Vaccaro AR, Hilibrand AS, Flynn JM, et al: Transcranial electric motor evoked potential monitoring during spine surgery: is it safe? Spine (Phila Pa 1976) 36:1046-1049, 2011

9. Skaggs DL, Lee C, Myung KS: Neuromonitoring changes are common and reversible with temporary internal distraction for severe scoliosis. Spine Deform 2:61-69, 2014

10. Skinner S, Sala F: Communication and collaboration in spine neuromonitoring: time to expect more, a lot more, from the neurophysiologists. J Neurosurg Spine 27:1-6, 2017

11. Skinner SA, Holdefer RN: Intraoperative neuromonitoring alerts that reverse with intervention: treatment paradox and what to do about it. J Clin Neurophysiol 31:118-126, 2014

12. Stecker MM: A review of intraoperative monitoring for spinal surgery. Surg Neurol Int 3 (Suppl 3):S174-S187, 2012

13. Vitale MG, Skaggs DL, Pace GI, Wright ML, Matsumoto H, Anderson RC, et al: Best practices in intraoperative neuromonitoring in spine deformity surgery: development of an intraoperative checklist to optimize response. Spine Deform 2:333-339, 2014

\section{Disclosures}

Dr. Hwang reports being on the speaker's bureau of Zimmer Biomet. Dr. Pahys reports being a consultant for DePuy Synthes, Globus Medical, and Zimmer Biomet. Dr. Samdani reports being a consultant for DePuy Synthes Spine, Ethicon, Globus Medical, Misonix, Stryker, and Zimmer Biomet.

\section{Author Contributions}

Conception and design: J Zuccaro, M Zuccaro. Acquisition of data: J Zuccaro, M Zuccaro. Analysis and interpretation of data: J Zuccaro, M Zuccaro. Drafting the article: J Zuccaro, M Zuccaro. Critically revising the article: all authors. Reviewed submitted version of manuscript: all authors. Approved the final version of the manuscript on behalf of all authors: J Zuccaro. Statistical analysis: J Zuccaro, M Zuccaro. Study supervision: J Zuccaro.

\section{Correspondence}

James Zuccaro, Shriners Hospitals for Children-Philadelphia, 3551 N Broad St., Philadelphia, PA 19140. email: docrun@ me.com. 